\title{
O NOVO CORONAVÍRUS E AS CONSEQUÊNCIAS NA SAÚDE MENTAL DOS PROFISSIONAIS DE SAÚDE ENVOLVIDOS EM SEU ENFRENTAMENTO
}

\author{
THE NEW CORONAVIRUS AND THE CONSEQUENCES IN THE MENTAL HEALTH OF HEALTH PROFESSIONALS \\ INVOLVED IN THEIR COURSE \\ EL NUEVO CORONAVIRUS Y LAS CONSECUENCIAS EN LA SALUD MENTAL DE LOS PROFESIONALES DE LA \\ SALUD INVOLUCRADOS EN SU AFRONTAMIENTO
}

\section{Érika da Silva Maciel}

Pós-doutorado em Ciência pelo Departamento de Clínica Médica (FCM-USP). Docente da Universidade Federal do Tocantins (UFT), Miracema, Tocantins. erikasmaciel@gmail.com.

\section{0-0002-9836-7665}

\section{Marcele Gotardelo}

Mestranda. Programa de Pós-graduação em Ensino em Ciências e Saúde PPGECS da Universidade Federal do Tocantins (UFT). Pós-graduada em Terapia Intensiva e Metodologias Ativas e Práticas Inovadoras. Graduação em Enfermagem. marcelegotardelo@gmail.com.

\section{0-0001-7179-7537}

\section{Allana Lima Moreira Rodrigues}

Mestranda. Programa de Pós-graduação em Ensino em Ciências e Saúde PPGECS da Universidade Federal do Tocantins (UFT). Pós-graduada em Saúde Coletiva. Graduação em Enfermagem. lana.moreiira@gmail.com.

\section{0-0001-8036-7280}

\section{Fernando Rodrigues Peixoto Quaresma}

Doutor em Ciências da Saúde (FMABC). Docente no curso de Enfermagem da Universidade Federal do Tocantins (UFT) e do Programa de Pós Graduação em Ensino em Ciências e Saúde PPGECS da Universidade Federal do Tocantins (UFT). ferodriguesto@gmail.com.

0000-0001-8407-0310

\section{Aldair Martins Barasuol}

Mestre em Ensino Ciências e Saúde pela Universidade Federal do Tocantins (UFT), Palmas/TO. Graduação em Enfermagem. aldair.barasuol@mail.uft.edu.br.

0000-0002-7122-4648

\section{Ladislau Ribeiro do Nascimento}

Doutor em Psicologia Social pela Universidade de São Paulo (USP). Docente do Curso de Psicologia e do Programa de Pós-Graduação em Ensino em Ciências e Saúde da Universidade Federal do Tocantins (UFT). ladislaunascimento@uft.edu.br.

\section{0-0002-6980-706X}

Correspondência: Universidade Federal do Tocantins, Coordenação de Enfermagem. Avenida NS 15, Quadra 109 Norte Plano Diretor Norte 77000000 - Palmas, TO - Brasil - Caixa-postal: 77001090

Recebido em: 12.10.2020.

Aceito em: 20.11.2020.

Publicado em: 01.01.2021.

\begin{abstract}
RESUMO:
A pandemia da doença causada pelo novo Coronavírus - SARS-Cov-2 se caracteriza como um dos maiores problemas de saúde pública internacional das últimas décadas. Este estudo se propôs a realizar uma revisão narrativa das evidências descritas sobre o impacto na saúde mental dos profissionais de saúde que atuam na prática assistencial relacionada à Covid-19. Os artigos evidenciaram que os problemas enfrentados pelos profissionais de saúde durante a pandemia podem levar à exaustão da equipe, esgotamento físico e mental contribuindo para o desenvolvimento de problemas psicológicos.
\end{abstract}

PALAVRAS-CHAVES: Covid-19; Saúde Mental; Profissionais de saúde.

\section{Introdução}

A Covid-19, doença causada pelo novo Coronavírus (SARS-Cov-2), foi inicialmente identificada em dezembro de 2019 na cidade de Wuhan, capital da província da China Central. O vírus possui um alto potencial de contágio e incidência exponencial e sua transmissão universalizada foi reconhecida pela Organização Mundial da Saúde (OMS) como uma pandemia (VASCONCELOS et al., 2020). A pandemia da COVID-19 se caracteriza como um dos maiores problemas de saúde pública internacional das últimas 
décadas, atingindo todo o mundo em uma velocidade nunca vista anteriomente (WHO, 2020).

Os sintomas da doença frequentemente envolvem tosse, febre e dificuldades respiratórias que podem levar à morte. O considerável número de casos que necessitam de intervenção hospitalar, incluindo cuidados em unidade de terapia intensiva, bem como a ausência de tratamento farmacológico eficaz e seguro, têm gerado grande preocupação sobre a crise dos sistemas de saúde em diferentes países (SCHMIDT et al., 2020).

A doença tem trazido um profundo impacto global, tornando-se a síndrome respiratória viral mais severa desde a pandemia de H1N1 (gripe espanhola), em 1918, assim, constituindo-se uma emergência de saúde pública de interesse internacional pela Organização Mundial da Saúde (OMS) (ORNELL et al., 2020b).

A quantidade de casos novos e de óbitos decorrentes do Coronavírus é impossível de ser expressa com exatidão, pois o seu crescimento acontece de forma exponencial, podendo levar ao esgotamento de leitos hospitalares em Unidades de Terapia Intensiva (UTI) e ao colapso dos serviços de saúde no mundo todo (ZHOU et al., 2020). No Brasil, as taxas encontradas no início de junho de 2020 já colocavam o país no segundo lugar em relação ao número de infectados, ficando atrás apenas dos Estados Unidos da América (EUA). O número de acometidos pela enfermidade cresce a cada dia, sem perspectivas científicas que vislumbrem seu fim (BRASIL, 2020; BRUNA-ROMERO; CARCIOFI, 2020; HOLANDA, 2020; ZHOU et al., 2020).

O elevado número de infectados e a taxa de mortalidade trouxeram insegurança e medo para população ocasionando consequências psicológicas e sociais que afetam a capacidade de mobilização de toda a sociedade. A situação acentuou-se devido à insuficiência dos mecanismos de controle (BROOKS et al., 2020).

Essas incertezas trazem problemas em diversas áreas, implicando diretamente no cotidiano e na saúde mental dos indivíduos, bem como dos profissionais de saúde que estão trabalhando direta ou indiretamente no enfrentamento da doença (BROOKS et al., 2020; MIRANDA et al., 2020; SCHMIDT et al., 2020a). Além do medo e da sensação de insegurança, destacam-se outras manifestações de adoecimento mental e de sofrimento psicossocial, tais como episódios depressivos, ansiedade, alterações do sono, intensificação de conflitos interpessoais, transtornos do pânico, raiva, insônia, abuso de álcool e outras drogas, ideações suicidas, suicídios e estresse (DUAN; ZHU, 2020).

A inexistência de uma vacina contra o novo Coronavírus tem reforçado entre os profissionais da saúde a importância da adoção de medidas de prevenção contra a infecção, como o uso de Equipamentos de Proteção Individual (EPI) e a higienização 
constante das mãos na assistência aos pacientes infectados ou não pelo vírus, a fim de reduzir os impactos negativos desta situação (HOLANDA, 2020).

Associado a isso, a pandemia traz consigo um problema extra para a saúde mental de médicos, enfermeiros e técnicos de enfermagem. Por atuarem diretamente no cuidado dos pacientes e estarem sob pressão, estes profissionais mostram-se vulneráveis ao adoecimento mental, devido à incansável e incessante luta contra a doença, permitindo a manifestação de transtornos associados a medo, estresse e ansiedade. Além disso, muitos profissionais de saúde sofrem em decorrência de preconceito e estigmatização associados ao fato de lidarem cotidianamente com pessoas infectadas ou que estão sob a suspeita de infecção (SCHMIDT. B. et al., 2020).

Assim, o objetivo deste estudo foi realizar uma revisão narrativa das evidências descritas sobre quais as consequências desta pandemia na saúde mental dos profissionais de saúde que atuam no enfrentamento do novo Coronavírus, a fim de elencar os principais problemas encontrados.

\section{Materiais e métodos}

O presente estudo é de caráter descritivo por meio de revisão narrativa da literatura.

As bases de dados utilizadas para a busca de artigos foram: Scientific Electronic Library Online (Scielo), Literatura Latino-Americana e do Caribe em Ciências de Saúde (LILACS) e PubMed ${ }^{\circledR}$ - National Library of Medicine (NIH). A pesquisa foi mediada pelos seguintes Descritores em Ciências da Saúde (DeCS): "Coronavírus", "saúde mental" e "profissionais da saúde" em português e "coronavirus", "mental health" e "health professional", em inglês.

Consideraram-se os artigos originais publicados em estudos primários durante o ano de 2020 realizados com profissionais de saúde. Resumos, recomendações e relatos de experiência foram excluídos do estudo, bem como aqueles encontrados em duplicidade nas bases de dados consultadas. Os títulos e os resumos de todos os artigos identificados na busca eletrônica foram revisados. Os estudos elegíveis segundo os critérios descritos foram obtidos integralmente e, com base nesta ação, elaborou-se uma lista de artigos para análise.

Foram identificados 69 resumos nas bases de dados. Após a leitura dos títulos e resumos foram excluídos 52 estudos, perfazendo o total de 14 artigos revisados na íntegra (Figura 1).

Figura 1 Fluxograma contendo o processo de seleção dos estudos. 


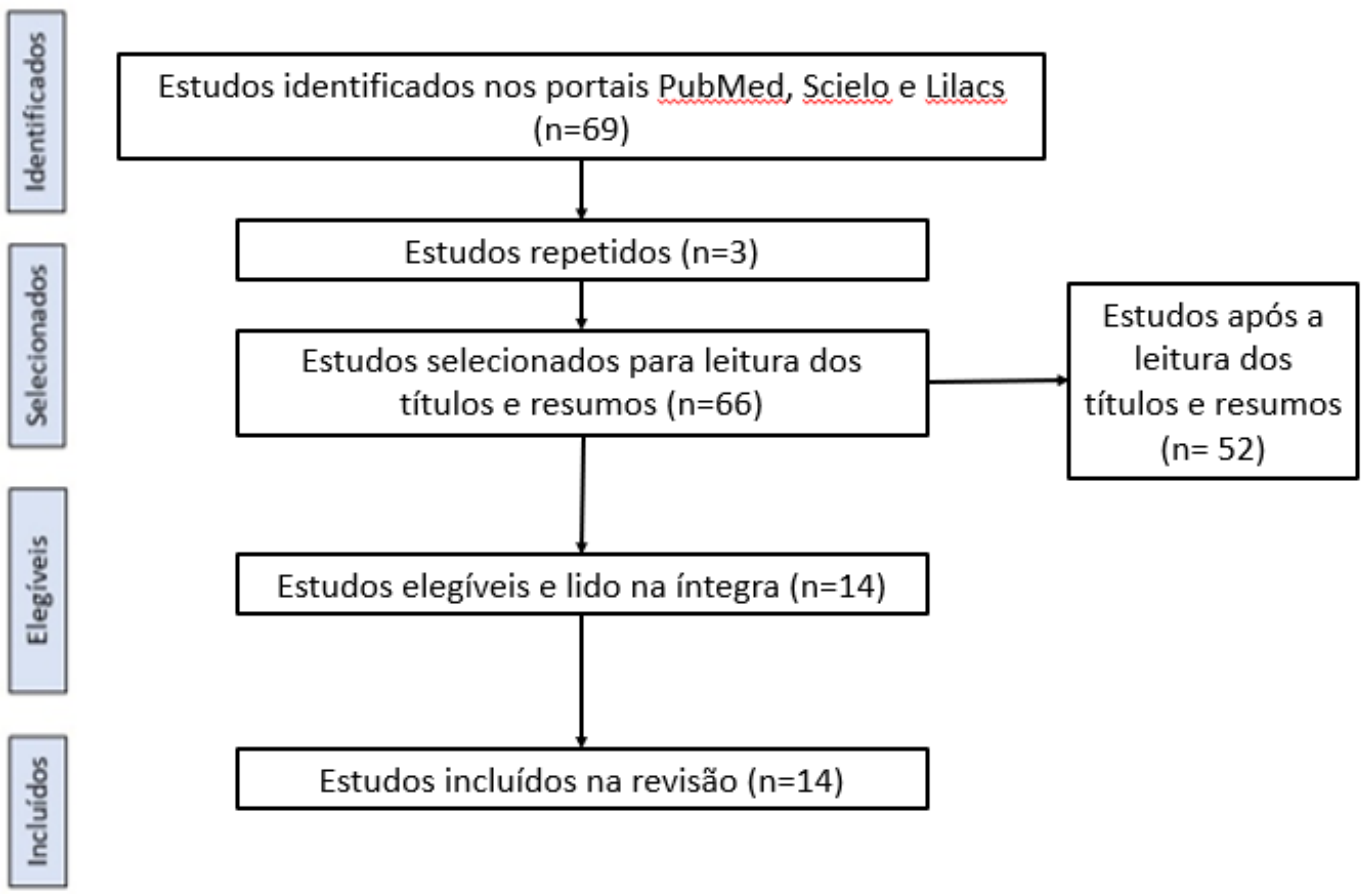

O Quadro 1 apresenta as principais características dos artigos que trazem resultados de estudos sobre o impacto da pandemia do novo Coronavírus na saúde mental de profissionais da saúde. Dos 14 trabalhos selecionados, 13 foram encontrados na língua inglesa e traduzidos para o português.

\section{Resultados}

Os estudos incluídos possuem, em sua maioria, desenhos de revisão da literatura, alguns com metanálise, há também estudos com dados primários, e os países de origem do estudo são Alemanha, Brasil, Canadá, China, Estados Unidos, Inglaterra, Itália e Irlanda. Os estudos analisados demonstraram diversas respostas psicológicas relacionadas à quarentena e ao enfrentamento da pandemia, incluindo ansiedade, estresse, depressão, insônia, medo e distúrbio psicótico. (Quadro 1)

Quadro 1 Autor, título segundo o idioma em que foi publicado, tipo de estudo e principais resultados.

\begin{tabular}{l|c|c|c} 
AUTOR & TíTULO & TIPO DE ESTUDO & PRINCIPAIS RESULTADOS \\
\hline
\end{tabular}




\begin{tabular}{|c|c|c|c|}
\hline $\begin{array}{l}\text { NETO, M. R. } \\
\text { L. et al. } 2020 .\end{array}$ & $\begin{array}{l}\text { When health } \\
\text { professionals } \\
\text { look death in the } \\
\text { eye: the mental } \\
\text { health of } \\
\text { professionals } \\
\text { who deal daily } \\
\text { with the } 2019 \\
\text { coronavirus } \\
\text { outbreak. }\end{array}$ & $\begin{array}{l}\text { Revisão nas bases de } \\
\text { dados eletrônicas } \\
\text { Scopus e Embase. }\end{array}$ & $\begin{array}{l}\text { Os autores descrevem vários sintomas e doenças } \\
\text { psiquiátricas observadas em profissionais de saúde a } \\
\text { partir de uma coletânea de artigos. }\end{array}$ \\
\hline $\begin{array}{l}\text { EL-HAGE, W. } \\
\text { et al. } 2020 .\end{array}$ & $\begin{array}{l}\text { Health } \\
\text { professionals } \\
\text { facing the } \\
\text { coronavirus } \\
\text { disease } 2019 \\
\text { (COVID-19) } \\
\text { pandemic: What } \\
\text { are the mental } \\
\text { health risks? }\end{array}$ & $\begin{array}{l}\text { Revisão de literatura } \\
\text { internacional, } \\
\text { levando em } \\
\text { consideração dados } \\
\text { de epidemias } \\
\text { anteriores (SARS- } \\
\text { CoV-1, H1N1) e } \\
\text { dados mais recentes } \\
\text { sobre o COVID-19. }\end{array}$ & $\begin{array}{l}\text { Os autores relatam que os fatores organizacionais } \\
\text { podem ser uma fonte de estresse: falta de } \\
\text { equipamento de proteção individual, falta de } \\
\text { comunicação, falta de materiais de atendimento, } \\
\text { interrupção do cotidiano familiar e social. Outros } \\
\text { fatores de risco são identificados como falta de apoio, } \\
\text { medo de infectar um ente querido, isolamento ou } \\
\text { estigma social e alto nível de estresse no trabalho. }\end{array}$ \\
\hline $\begin{array}{l}\text { ROHR, S. et } \\
\text { al. } 2020\end{array}$ & $\begin{array}{l}\text { Psychosocial } \\
\text { Impact of } \\
\text { Quarantine } \\
\text { Measures During } \\
\text { Serious } \\
\text { Coronavirus } \\
\text { Outbreaks: A } \\
\text { Rapid Review } \\
\end{array}$ & $\begin{array}{l}\text { Revisão de literatura } \\
\text { para estudos } \\
\text { relacionados a surtos } \\
\text { de SARS-CoV e } \\
\text { MERS-CoV. }\end{array}$ & $\begin{array}{l}\text { As medidas de quarentena durante surtos graves de } \\
\text { coronavírus têm extensas consequências negativas } \\
\text { para a saúde mental. As abordagens de prevenção e } \\
\text { intervenção para atenuar o impacto psicossocial } \\
\text { devem ser um componente integral da resposta a } \\
\text { crises durante condições de pandemia. }\end{array}$ \\
\hline $\begin{array}{l}\text { SIMONELLI, } \\
\text { C. et al. } 2020\end{array}$ & $\begin{array}{l}\text { The } \\
\text { psychological } \\
\text { impact of a } \\
\text { pandemic } \\
\text { outbreak on } \\
\text { medical staff - } \\
\text { Literature review }\end{array}$ & $\begin{array}{l}\text { Revisão de literatura } \\
\text { sobre o impacto } \\
\text { psicológico de um } \\
\text { surto de pandemia } \\
\text { na equipe médica. }\end{array}$ & $\begin{array}{l}\text { Os autores descrevem que as evidências do impacto } \\
\text { a curto e longo prazo da epidemia na saúde mental } \\
\text { das equipes médicas. Exposição direta a pacientes } \\
\text { afetados, risco de contágio, percepção subjetiva de } \\
\text { sua condição médica como estar isolado ou em } \\
\text { quarentena, todos constituem fatores de risco para } \\
\text { sintomas mentais, como depressão, ansiedade e pós- } \\
\text { trauma. }\end{array}$ \\
\hline $\begin{array}{l}\text { BOHLKEN, J. } \\
\text { et al. } 2020\end{array}$ & $\begin{array}{l}\text { COVID-19 } \\
\text { Pandemic: Stress } \\
\text { Experience of } \\
\text { Healthcare } \\
\text { Workers - A Short } \\
\text { Current Review }\end{array}$ & $\begin{array}{l}\text { Revisão bibliográfica } \\
\text { utilizando os termos } \\
\text { "COVID-19", } \\
\text { "estresse", "saúde } \\
\text { mental", } \\
\text { "profissional de } \\
\text { saúde", "equipe", } \\
\text { "psiquiatria". }\end{array}$ & $\begin{array}{l}\text { O Questionário de Saúde do Paciente } 9 \text { (PHQ9), a } \\
\text { Escala de Autoavaliação de Ansiedade (SAS) e a Escala } \\
\text { de Impacto de Eventos (IES-R) foram os instrumentos } \\
\text { de teste utilizados. Foi relatada uma tensão extensa } \\
\text { devido à experiência de estresse, bem como a } \\
\text { sintomas de depressão e ansiedade. A gravidade dos } \\
\text { sintomas mentais foi influenciada por idade, sexo, } \\
\text { ocupação, especialização, tipo de atividades } \\
\text { realizadas e proximidade com os pacientes COVID-19. }\end{array}$ \\
\hline $\begin{array}{l}\text { KISELY, L. et } \\
\text { al. } 2020\end{array}$ & $\begin{array}{l}\text { Occurrence, } \\
\text { prevention, and } \\
\text { management of } \\
\text { the psychological }\end{array}$ & $\begin{array}{l}\text { Revisão } \\
\text { metanálise. }\end{array}$ & $\begin{array}{l}\text { A equipe em contato com os pacientes afetados } \\
\text { apresentou maiores níveis de estresse agudo ou pós- } \\
\text { traumático e sofrimento psicológico. Os fatores de } \\
\text { risco para sofrimento psíquico incluem ser mais }\end{array}$ \\
\hline
\end{tabular}




\begin{tabular}{|c|c|c|c|}
\hline & $\begin{array}{lr}\text { effects } & \text { of } \\
\text { emerging } & \text { virus } \\
\text { outbreaks } & \text { on } \\
\text { healthcare } & \\
\text { workers: rapid } & \\
\text { review and } & \text { meta- } \\
\text { analysis. } & \end{array}$ & & $\begin{array}{l}\text { jovem, ser mais jovem, ser pai de filhos dependentes } \\
\text { ou ter um membro da família infectado. Quarentena } \\
\text { mais longa, falta de apoio prático e estigma também } \\
\text { contribuíram. Comunicação clara, acesso a proteção } \\
\text { pessoal adequada, descanso adequado e apoio } \\
\text { prático e psicológico foram associados à redução da } \\
\text { morbidade. }\end{array}$ \\
\hline $\begin{array}{l}\text { ORNELL, F. } \\
\text { et al. } 2020\end{array}$ & $\begin{array}{l}\text { The impact of the } \\
\text { COVID-19 } \\
\text { pandemic on the } \\
\text { mental health of } \\
\text { healthcare } \\
\text { professionals. }\end{array}$ & Revisão de literatura. & $\begin{array}{l}\text { Os autores abordam as consequências observadas } \\
\text { após as últimas pandemias (SARS e MERS) e destacam } \\
\text { conceitos como o trauma vicário ou estresse } \\
\text { traumático secundário, além de destacarem o uso de } \\
\text { instrumentos de rastreamento e intervenções para o } \\
\text { cuidado psicológico/psiquiátrico. }\end{array}$ \\
\hline $\begin{array}{l}\text { SCHMIDT, B } \\
\text { et al, } 2020\end{array}$ & $\begin{array}{l}\text { Saúde mental e } \\
\text { intervenções } \\
\text { psicológicas } \\
\text { diante da } \\
\text { pandemia do } \\
\text { novo Coronavírus } \\
\text { (COVID-19) }\end{array}$ & Revisão de literatura. & $\begin{array}{l}\text { Os autores descrevem aspectos emocionais e } \\
\text { intervenções psicoterapêuticas voltadas à população } \\
\text { em geral e aos profissionais de saúde no contexto da } \\
\text { pandemia. }\end{array}$ \\
\hline $\begin{array}{l}\text { PETZOL, M. } \\
\text { B.; PLAG, J.; } \\
\text { STROLE, A. } \\
2020\end{array}$ & $\begin{array}{l}\text { Dealing with } \\
\text { psychological } \\
\text { distress by } \\
\text { healthcare } \\
\text { professionals } \\
\text { during the } \\
\text { COVID-19 } \\
\text { pandemia }\end{array}$ & $\begin{array}{l}\text { O artigo resume as } \\
\text { recomendações da } \\
\text { OMS e do Comitê } \\
\text { Permanente entre } \\
\text { Agências das Nações } \\
\text { Unidas (IASC). }\end{array}$ & $\begin{array}{l}\text { O artigo fornece as primeiras indicações do que os } \\
\text { profissionais de saúde e seus gestores podem fazer } \\
\text { para reduzir o estresse psicológico. O controle do } \\
\text { estresse e das emoções violentas, o apoio social, a } \\
\text { manutenção das necessidades básicas e o } \\
\text { autocuidado desempenham um papel central. }\end{array}$ \\
\hline $\begin{array}{l}\text { HUANG, J. Z. } \\
\text { et al. } 2020\end{array}$ & $\begin{array}{l}\text { Mental health } \\
\text { survey of medical } \\
\text { staff in a tertiary } \\
\text { infectious disease } \\
\text { hospital for } \\
\text { COVID-19 }\end{array}$ & $\begin{array}{l}\text { Estudo transversal } \\
\text { por amostragem por } \\
\text { Cluster }\end{array}$ & $\begin{array}{l}\text { Na epidemia de COVID-19, a incidência de transtorno } \\
\text { de ansiedade e estresse é alta entre a equipe médica. } \\
\text { As instituições médicas devem fortalecer o } \\
\text { treinamento de habilidades psicológicas da equipe } \\
\text { médica. Atenção especial deve ser dada à saúde } \\
\text { mental das enfermeiras. }\end{array}$ \\
\hline $\begin{array}{l}\text { LAl, J. et al. } \\
2020\end{array}$ & 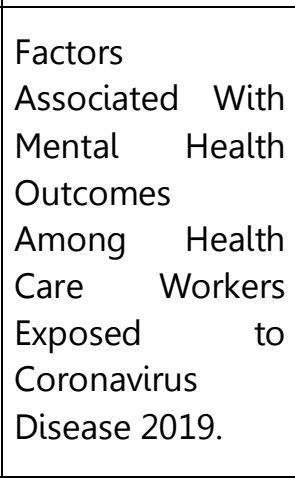 & $\begin{array}{lrr}\text { Estudo } & \text { transversal, } \\
\text { estratificado } & \text { por } \\
\text { região, } & \text { foram } \\
\text { coletados } & \text { dados } \\
\text { demográficos } & \text { e } \\
\text { medições } & \text { de } & \text { saúde } \\
\text { mental } & \text { de } & 1257 \\
\text { profissionais } & \text { de } \\
\text { saúde } & \text { em } & 34 \\
\text { hospitais } & \text { na } & \text { China. } \\
\end{array}$ & $\begin{array}{l}\text { Uma proporção considerável de profissionais de } \\
\text { saúde relatou apresentar sintomas de depressão, } \\
\text { ansiedade, insônia, e angústia, especialmente } \\
\text { mulheres, enfermeiras, profissionais de saúde de linha } \\
\text { direta envolvidos no diagnóstico, tratamento ou } \\
\text { prestação de cuidados de enfermagem a pacientes } \\
\text { com suspeita ou confirmação de COVID-19. }\end{array}$ \\
\hline $\begin{array}{l}\text { LU, W. } \\
\text { WANG, H. } \\
\text { LIN, W. LI, L. } \\
2020\end{array}$ & $\begin{array}{l}\text { Psychological } \\
\text { status of medical } \\
\text { workforce during } \\
\text { the COVID-19 } \\
\text { pandemic: A }\end{array}$ & $\begin{array}{l}\text { Pesquisa transversal } \\
\text { de um centro } \\
\text { médico por meio de } \\
\text { questionários on- } \\
\text { line. }\end{array}$ & $\begin{array}{l}\text { Os autores relatam que em comparação com a equipe } \\
\text { não clínica, a equipe médica da linha de frente com } \\
\text { contato próximo com pacientes infectados, inclusive } \\
\text { trabalhando nos departamentos de doenças } \\
\text { respiratórias, de emergência, infecciosas e UTI, }\end{array}$ \\
\hline
\end{tabular}




\begin{tabular}{|c|c|c|c|}
\hline & $\begin{array}{l}\text { cross-sectional } \\
\text { study. }\end{array}$ & & $\begin{array}{l}\text { apresentou pontuações mais altas na escala de medo, } \\
\text { HAMA e HAMD, e era } 1,4 \text { vezes mais propensa a sentir } \\
\text { medo, duas vezes mais propensos a sofrer ansiedade } \\
\text { e depressão. }\end{array}$ \\
\hline $\begin{array}{l}X U \text {, J. et al. } \\
2020\end{array}$ & $\begin{array}{l}\text { Psychological } \\
\text { status of surgical } \\
\text { staff during the } \\
\text { COVID-19 } \\
\text { outbreak. }\end{array}$ & Caso-controle. & $\begin{array}{l}\text { Os resultados mostraram, utilizando } 4 \text { (quatro) } \\
\text { escalas aplicadas a dois grupos de profissionais de } \\
\text { saúde, que os níveis de ansiedade e de depressão da } \\
\text { equipe cirúrgica durante o período do surto foram } \\
\text { significativamente maiores no período da pandemia, } \\
\text { merecendo, intervenções psicológicas. }\end{array}$ \\
\hline $\begin{array}{l}\text { ANMELLA, } \\
\text { G. et al. } 2020\end{array}$ & $\begin{array}{l}\text { Unravelling } \\
\text { potential severe } \\
\text { psychiatric } \\
\text { repercussions on } \\
\text { healthcare } \\
\text { professionals } \\
\text { during the } \\
\text { COVID-19 crisis. }\end{array}$ & $\begin{array}{l}\text { Seção: } \\
\text { correspondência. } \\
\text { Desenho: caso } \\
\text { clínico. }\end{array}$ & $\begin{array}{l}\text { Os autores relatam o caso de um médico clínico geral } \\
\text { sem histórico psiquiátrico que desenvolveu agitação } \\
\text { psicomotora e surto psicótico reativo a circunstâncias } \\
\text { relacionadas à Covid-19. }\end{array}$ \\
\hline
\end{tabular}

Dentre os problemas de saúde mental encontrados, foi identificada a presença de trauma vicário, que é o fenômeno no qual os profissionais de saúde experimentam sintomas semelhantes aos dos pacientes devido à exposição contínua. O trauma indireto, comum durante grandes catástrofes, também foi observado, manifestando-se por perda de apetite, fadiga, declínio físico, distúrbios do sono e da atenção, irritabilidade, dormência, medo e desespero (ORNELL et al., 2020a; SCHMIDT et al., 2020b).

Identificaram-se diversos fatores potencialmente geradores de sofrimento psicológico relacionados ao enfrentamento da pandemia, dentre estes, a incerteza sobre a duração da crise, inexistência de terapias comprovadas ou vacina e potencial escassez de recursos de saúde, incluindo equipamentos de proteção individual (EL-HAGE et al., 2020; WU; STYRA; GOLD, 2020).

Os profissionais de saúde também se mostraram angustiados com os efeitos do distanciamento social em relação ao desejo de estarem presentes junto a suas famílias e à possibilidade de transmitir a doença a amigos e familiares (EL-HAGE et al., 2020; WU; STYRA; GOLD, 2020).

Além disso, a exposição direta a pacientes afetados, o risco de contágio, a percepção subjetivamente negativa da condição médica e o fato de estarem isolados ou em quarentena representaram fatores de risco para a ocorrência de sintomas mentais (RÖHR et al., 2020).

Há ainda a presença de outra forma de sofrimento psíquico, a lesão ou dano moral, onde o profissional possui pensamentos negativos sobre si ou sobre os outros, como 
sentimentos intensos de vergonha, culpa ou nojo (CHEN et al., 2020; GREENBERG et al., 2020).

Fatores organizacionais e estruturais também foram descritos, tais como o esgotamento dos equipamentos de proteção individual, a falta de medicamentos específicos, as preocupações sobre a impossibilidade de prestar cuidados competentes se implantados em uma nova área, a falta de acesso a informações e comunicações atualizadas, a preocupação com a mudança rápida de informações e a escassez de ventiladores e leitos de unidades de terapia intensiva necessários para o atendimento de pacientes críticos (EL-HAGE et al., 2020).

\section{Discussão}

Durante a pandemia, o mundo passa pela desaceleração das atividades cotidianas e as pessoas são incentivadas a praticarem o distanciamento social com o intuito de reduzir o contato entre os indivíduos, diminuindo consequentemente novas infecções, porém, os profissionais de saúde seguem na direção oposta (VASCONCELOS et al., 2020).

Devido ao aumento excepcional da procura pelos serviços de saúde, estes profissionais vêm enfrentando longos e intensos turnos de trabalho, muitas vezes com recursos escassos, infraestrutura inapropriada e com a necessidade do uso dos equipamentos de proteção individual (EPI) que geralmente são racionados e também causam desconforto físico (MIRANDA et al., 2020; SOARES et al., 2020). Essa problemática impacta diretamente no risco de contágio, que se eleva na assistência direta aos infectados (HELIOTERIO et al., 2020).

Segundo dados do Conselho Federal de Enfermagem (COFEN), cerca de 630 profissionais de enfermagem haviam ido a óbito pelo Coronavírus em todo o mundo, e o Brasil já totalizava 98 desses óbitos no início de junho, ultrapassando os EUA, então com 91 falecimentos (COFEN, 2020). Na Itália, o número de profissionais infectados chegava a 4.884 casos, tendo sido registrado um total de 24 óbitos de médicos (HELIOTERIO et al., 2020).

Estes dados refletem a vulnerabilidade desses profissionais, que por muitas vezes sentem-se despreparados para realizar procedimentos e intervenções clínicas aos pacientes infectados, devido à mudança contínua de protocolos e tratamentos, que até o momento em que se produziu este artigo não estavam bem estabelecidos (MIRANDA et al., 2020; ORNELL et al., 2020b).

Condições precárias de trabalho, escassez de recursos e materiais, bem como problemas nos fluxos e organização do serviço implicam em cargas de trabalho elevadas, aumento nas jornadas laborais e privação de pausas e repousos (HELIOTERIO et al., 
2020). Dificuldades intensificadas em um cenário de crise e que são constantemente apontadas nos serviços de saúde como fatores redutores de capacidade para o trabalho e geradores de problemas psicológicos nos profissionais da saúde (BROOKS et al., 2020; HELIOTERIO et al., 2020).

As implicações na saúde mental em consequência da pandemia do novo Coronavírus ainda são pouco estudadas, por se tratar de um evento recente, mas indicam impactos negativos sérios. Os efeitos psicológicos dessa pandemia nos profissionais de saúde não podem ser menosprezados.

Pesquisas realizadas anteriormente durante outras pandemias e surtos infecciosos, como Ebola, HIV, Zika e H1N1, apresentaram desdobramentos disruptivos do habitual, em curto, médio e longo prazo, para os profissionais de saúde e para a população em geral (ORNELL et al., 2020b; POWELL; FIELD, 2007).

Um exemplo a ser citado aconteceu na epidemia de Ebola no ano de 1995, quando sobreviventes revelaram constante medo de morrer, de contaminar outras pessoas, do abandono nas relações familiares e no ciclo de amizades, assim como temiam a estigmatização social. Os trabalhadores da área de saúde também fizeram menção medo de provocarem a própria infecção, além de relatarem medo de morrer e de transmitir a doença a seus familiares. Muitos manifestaram sentimento de desvalia, aflição por estarem afastados de suas casas e estresse (SCHMIDT et al, 2020).

O medo pode ser considerado uma forma de se defender em ocasiões de estresse ou de perigo. Ele é fundamental para preparar o organismo humano, tornando-o mais forte e vigilante. Entretanto, quando esse medo se torna crônico ou exagerado, pode comprometer a saúde psicológica, resultando em possíveis distúrbios psiquiátricos (ORNELL et al., 2020).

O apoio à família é protetor contra o estresse ocupacional, porém o profissional tem medo de se relacionar e infectar algum membro de sua família e a experiência do isolamento social, o distanciamento interpessoal são também dois fatores que possuem impactos psicológicos negativos ao profissional da saúde (GAVIN et al., 2020)

Um estudo avaliou o status psicológico comparando os valores médios de medo, ansiedade e depressão entre dois grupos, a equipe médica e a equipe administrativa de um hospital, por meio de um questionário online. De 2299 participantes, 2042 eram da equipe médica e 257 da equipe administrativa. A intensidade dos sintomas entre as equipes foram significativamente diferentes entre os dois grupos, onde a equipe que se encontrava em contato próximo a pacientes infectados possuía escores ainda maiores, tornando-os mais suscetíveis a distúrbios psicológicos (LU et al., 2020). 
A exposição a mortes em larga escala, assim como a decepção ao não conseguir salvar todas as vidas e até mesmo pressões, ameaças e agressões vindas de pacientes que não conseguem assistência devido ao déficit de recursos físicos e materiais, ou em razão de outros fatores, expõem ainda mais os profissionais de saúde ao adoecimento mental, tornando-os cada vez mais ansiosos e vulneráveis a outras patologias (ORNELL et al., 2020; TAYLOR, 2019).

Estudo realizado na China evidenciou que muitos profissionais de saúde que trabalham na linha de frente no combate ao Coronavírus apresentaram dados importantes relacionados à saúde mental. Em um total de 1257 profissionais de 34 hospitais (61\% enfermeiros e $39 \%$ médicos), cerca de $50 \%$ sofriam com sintomas de depressão, 45\% de ansiedade, 34\% insônia e 72\% de angústia. Carga de trabalho excessiva, discriminação e isolamento foram apontados como agravantes do sofrimento psíquico, deixando esses trabalhadores mais vulneráveis aos efeitos de exaustão física e psíquica em associação com medo, distúrbios emocionais e problemas de sono (SAIDEL et al., 2020).

Pesquisas realizadas na Alemanha identificaram agravantes associados aos profissionais, sendo eles: medo do risco de infectar a si e aos outros; não ter clareza do diagnóstico, pelo fato de os sintomas do COVID-19 aparentarem os de outras doenças; ter contato com os familiares; além da preocupação com a saúde física e mental, principalmente no caso de profissionais que possuíam um transtorno mental préexistente (PETZOLD et al, 2020).

A sobrecarga da assistência ofertada ao paciente diagnosticado com COVID-19 também desponta como um fator capaz de levar equipes à exaustão, provocando esgotamento físico e mental. Profissionais de saúde infectados com o vírus podem sentir desamparo, desespero, desesperança, além de sofrerem as consequências do isolamento. Este cenário traz prejuízos à saúde mental desses indivíduos, precisando que sejam tomadas medidas protetoras e que sejam realizadas intervenções efetivas urgentes objetivando a promoção da saúde mental desses profissionais (SCHMIDT. B. et al., 2020).

A gravidade da pandemia pode desencadear exaustão emocional em profissionais que atuam junto aos pacientes infectados. Um estudo relata um maior risco de desenvolver transtorno de estresse pós-traumático (TEPT), observados também após o término da pandemia. Isto pode ocorrer devido à exposição contínua e com sintomas de distúrbio do sono, irritabilidade, perda de apetite, fadiga, medo, entre outros (LI et al., 2020). 
É inevitável que os profissionais da saúde, que estejam atuando na linha de frente, encontrem-se mais vulneráveis a desenvolver problemas emocionais, pois, além de tudo, lidam também com seus sentimentos de fracasso, impotência, estresse, incertezas sobre a nova doença, no que diz respeito ao tratamento e à dificuldade de superar o contato com perdas de seus pacientes (MIRANDA, et al, 2020).

Vale observar a possibilidade e a necessidade de profissionais de saúde observar em sinais de que a pandemia pode ter intensificado ou produzido um quadro de adoecimento mental. Os indícios são expressos através de persistência de sintomas, bem como intensidade do sofrimento, complicações no funcionamento social e cotidiano, aumento de conflitos familiares ou sociais, ou até mesmo ideações suicidas, dentre outras manifestações de sofrimento psíquico (BRASIL, 2020b).

A OMS recomenda a adoção de cuidados para o manejo do adoecimento mental e do sofrimento psicossocial em profissionais de saúde em tempos de pandemia. Dentre as recomendações, destacam-se as seguintes: (1) autocuidado, (2) busca de apoio a partir do contato com algum colega de confiança ou até mesmo um familiar, (3) reconhecimento e identificação dos próprios sentimentos, (4) resgate de estratégias utilizadas para a superação de adversidades em outros momentos de crise e de emergência em saúde (WHO, 2020).

A despeito da publicação oficial mencionada, os esforços das autoridades de saúde pública no decorrer das epidemias têm abrangido a compreensão dos efeitos físicos e biológicos que a doença pode trazer, dando pouca ênfase à saúde mental. Diante desse contexto não podemos minimizar as consequências psicológicas que este cenário tem causado sobre os profissionais de saúde em particular. Além das diversas complicações que circundam o processo de enfrentamento e controle de um surto pandêmico, é necessário assegurar aos profissionais de saúde um cuidado adequado em saúde mental, incluindo ações voltadas à redução do sofrimento mental ao longo desse problema de saúde pública (FARO et al., 2020).

Diante das evidências anteriormente descritas, verifica-se o impacto negativo da pandemia na saúde mental dos profissionais da saúde, influenciando diretamente em sensações como o medo, estresse e outros, que podem desencadear ou agravar transtornos mentais como os transtornos depressivos e os de ansiedade (EL-HAGE et al., 2020; HUANG et al., 2020; LU et al., 2020; NETO et al., 2020; XU et al., 2020).

\section{Considerações finais}

É consenso na literatura que, diante desse grave problema de saúde pública, os profissionais de saúde podem desencadear ou intensificar importantes sintomas 
psicológicos por estarem mais vulneráveis devido às dificuldades vivenciadas em seu trabalho.

Vários estudos relatam sobre métodos utilizados para melhorar a saúde mental dos profissionais da saúde, relata que existem diversas estratégias de cuidado à saúde mental, porém relatam a importância de implementar assertivamente essas ações.

Uma atenção da gestão organizacional deve ser feita para auxiliar o profissional com implementação dos serviços de intervenção psicológica. É necessária uma liderança forte com comunicação clara, honesta e aberta para compensar medos e incertezas, apoio à saúde mental aumentará a autoeficácia e a confiança individuais, enfatizar o altruísmo de trabalhar na assistência à saúde e servir ao bem maior ajudará os profissionais de saúde a serem lembrados de seus propósitos em tempos de crise.

Desta forma, destaca-se o papel fundamental das instituições empregadoras que devem se preocupar com a classe trabalhadora da saúde e oferecer cuidado e apoio psicológico, além de valorizar esses profissionais através de uma infraestrutura digna, com EPIs e reconhecimento ao seu trabalho.

\section{Referências}

BROOKS, S. K. et al. The psychological impact of quarantine and how to reduce it: rapid review of the evidenceThe LancetLancet Publishing Group, , mar. 2020.

BRUNA-ROMERO, O.; CARCIOFI, B. A. M. Estimativa da subnotificação de casos da covid19 no estado de santa catarina. Universidade Federal de Santa Catarina, p. 1-8, 2020.

CHEN, Q. et al. Mental health care for medical staff in China during the COVID-19 outbreakThe Lancet PsychiatryElsevier Ltd, , 1 abr. 2020. Disponível em: <https://pubmed.ncbi.nlm.nih.gov/32085839/>. Acesso em: 7 ago. 2020

COFEN. Brasil ultrapassa EUA em mortes de profissionais de Enfermagem por Covid-19 Conselho Federal de Enfermagem - Brasil.

COLE, C. et al. Adapting IAPT Services to Support Frontline NHS Staff during the Covid19 Pandemic: The Homerton Covid Psychological Support (HCPS) Pathway. Cognitive Behaviour Therapist, v. 13, n. 12, p. 1-12, 2020.

EL-HAGE, W. et al. Health professionals facing the coronavirus disease 2019 (COVID-19) pandemic: What are the mental health risks? Encephale, v. 46, n. 3S, 22 abr. 2020.

FARO, A. et al. COVID-19 e saúde mental: a emergência do cuidado. Estudos de Psicologia (Campinas), v. 37, 2020.

GAVIN, B. et al. Caring for the Psychological Well-Being of Healthcare Professionals in the Covid-19 Pandemic Crisis. Irish Medical Journal, v. 113, n. 4, p. 53, 2020.

GREENBERG, N. et al. Managing mental health challenges faced by healthcare workers during covid-19 pandemicThe BMJBMJ Publishing Group, , 26 mar. 2020. Disponível em: <https://pubmed.ncbi.nlm.nih.gov/32217624/>. Acesso em: 7 ago. 2020 
HELIOTERIO, M. C. et al. COVID-19: why is health protection for health workers a priority in combating the pandemic? Scientific Electronic Library Online, p. 1-18, 2020.

HOLANDA, V. N. PANDEMIA DE COVID-19 E OS ESFORÇOS DA CIÊNCIA PARA COMBATER O NOVO CORONAVÍRUS. Revista Interfaces: Saúde, Humanas e Tecnologia, v. 8, n. 1, p. 360-361, abr. 2020.

HUANG, J. Z. et al. Mental health survey of medical staff in a tertiary infectious disease hospital for COVID-19. Zhonghua lao dong wei sheng zhi ye bing za zhi = Zhonghua laodong weisheng zhiyebing zazhi $=$ Chinese journal of industrial hygiene and occupational diseases, v. 38, n. 3, p. 192-195, 20 mar. 2020.

$\mathrm{LI}$, Z. et al. Vicarious traumatization in the general public, members, and non-members of medical teams aiding in COVID-19 control. Brain, Behavior, and Immunity, v. 20, 2020.

LU, W. et al. Psychological status of medical workforce during the COVID-19 pandemic: A cross-sectional study. Psychiatry Research, v. 288, p. 1-5, 1 jun. 2020.

MIRANDA, F. M. D. et al. CONDIÇÕES DE TRABALHO E O IMPACTO NA SAÚDE DOS PROFISSIONAIS DE ENFERMAGEM FRENTE A COVID-19. Cogitare Enfermagem, v. 25, n. 0, maio 2020.

NETO, M. L. R. et al. When health professionals look death in the eye: the mental health of professionals who deal daily with the 2019 coronavirus outbreak. Psychiatry Research, v. 288, 1 jun. 2020.

ORNELL, F. et al. The impact of the COVID-19 pandemic on the mental health of healthcare professionals . Cadernos de Saúde Pública, v. 36, n. 4, p. 1-6, 2020a.

ORNELL, F. et al. "Pandemic fear" and COVID-19: mental health burden and strategies. Revista brasileira de psiquiatria (Sao Paulo, Brazil: 1999), v. 42, n. 3, p. 232-235, abr. 2020b.

POWELL, H.; FIELD, M. Stress increases attentional bias for alcohol cues in social drinkers who drink to cope. Alcohol and Alcoholism, v. 42, n. 6, p. 560-566, 2007.

RÖHR, S. et al. Psychosocial Impact of Quarantine Measures during Serious Coronavirus Outbreaks: A Rapid Review. Psychiatrische Praxis, v. 47, n. 4, p. 179-189, 1 maio 2020.

SAIDEL, M. G. B. et al. Intervenções em saúde mental para profissionais de saúde frente a pandemia de Coronavírus. Rev enferm UERJ, v. 28, n. 49923, 2020.

SCHMIDT. B. et al. Saúde mental e intervenções psicológicas diante da pandemia do novo coronavírus (COVID-19). Estudos de Psicologia, v. 37, p. 1-13, 2020.

SCHMIDT, B. et al. Impactos na Saúde Mental e Intervenções Psicológicas Diante da Pandemia do Novo Coronavírus (COVID-19). Revista Estudos de Psicologia (Campinas), v. 0, n. 1, p. 1-26, 2020 a.

SOARES, S. S. S. et al. Pandemia de Covid-19 e o uso racional de equipamentos de proteção individual. Revista Enfermagem UERJ, v. 28, n. 0, p. e50360, maio 2020.

VASCONCELOS, C. S. DA S. et al. O Novo Coronavírus e os impactos psicológicos da quarentena. DESAFIOS - Revista Interdisciplinar da Universidade Federal do Tocantins, v. 7, n. Especial-3, p. 75-80, abr. 2020.

WHO. Mental health and psychosocial considerations during the COVID-19 outbreakWorld Health Organization (WHO), 2020.

WU, P. E.; STYRA, R.; GOLD, W. L. Mitigating the psychological effects of COVID-19 on health care workers. CMAJ, v. 192, n. 17, p. 460, 27 abr. 2020. 


\section{Qrevisto \\ Observatório}

$X U$, J. et al. Psychological status of surgical staff during the COVID-19 outbreak. Psychiatry Research, v. 288, 1 jun. 2020.

ZHOU, F. et al. Clinical course and risk factors for mortality of adult inpatients with COVID-19 in Wuhan, China: a retrospective cohort study. The Lancet, v. 395, n. 10229, p. 1054-1062, mar. 2020.

\begin{abstract}
:
The disease pandemic caused by the new Coronavirus - SARS-Cov-2 is characterized as one of the biggest international public health problems in recent decades. This study aimed to perform a narrative review of the evidence described on the impact on mental health of health professionals who work in care practice related to Covid-19. The articles showed that the problems faced by health professionals during the pandemic can lead to exhaustion of the team, physical and mental exhaustion, contributing to the development of psychological problems.

KEYWORDS: Covid-19; Mental health; Health professionals.
\end{abstract}

\begin{abstract}
RESUMEN:
La pandemia de enfermedad causada por el nuevo coronavirus - SARS-Cov-2 se caracteriza por ser uno de los mayores problemas de salud pública internacional en las últimas décadas. Este estudio tuvo como objetivo realizar una revisión narrativa de la evidencia descrita sobre el impacto en la salud mental de los profesionales de la salud que trabajan en la práctica asistencial relacionada con Covid-19. Los artículos mostraron que los problemas que enfrentan los profesionales de la salud durante la pandemia pueden llevar al agotamiento del equipo, al agotamiento físico y mental, contribuyendo al desarrollo de problemas psicológicos.
\end{abstract}

PALABRAS-CLAVES: COVID-19; Salud mental; Profesionales de la salud. 